\title{
Effect of Emulsifier Concentration on the Oxidation of an 0/W Emulsion Prepared from Canola Oil
}

\author{
Yukihiro Yamamoto*, Rino Misawa \\ Faculty of Life and Environmental Sciences, Prefectural University of Hiroshima, Shobara, Japan \\ Email: *yyamamoto@pu-hiroshima.ac.jp
}

How to cite this paper: Yamamoto, Y. and Misawa, R. (2018) Effect of Emulsifier Concentration on the Oxidation of an $\mathrm{O} / \mathrm{W}$ Emulsion Prepared from Canola Oil. Food and Nutrition Sciences, 9, 683-692. https://doi.org/10.4236/fns.2018.96052

Received: May 23, 2018

Accepted: June 24, 2018

Published: June 27, 2018

Copyright (c) 2018 by authors and Scientific Research Publishing Inc. This work is licensed under the Creative Commons Attribution International License (CC BY 4.0).

http://creativecommons.org/licenses/by/4.0/

\section{(c) (i) Open Access}

\begin{abstract}
Oils and fats exist in many foods as emulsions, and preventing the oxidation of emulsion is vital importance for the food industry. In this study, the effect of different concentrations of Tween $20(0.5,2.5,5.0,10 \mathrm{w} \%$ vs. oil) on the oxidation of canola oil $\mathrm{O} / \mathrm{W}$ emulsion was investigated. Oxidation was conducted at $34^{\circ} \mathrm{C}$ in the dark and accelerated using 2,2-azobis (2,4-dimethylvaleronitrile) (AMVN) and 2,2'-azobis (2-methylpropionamidine) dihydrochloride (AAPH) as radical generators. Peroxide value $(\mathrm{PV}, \mathrm{mEq} / \mathrm{kg})$ was monitored as generation of the primary oxidation compound. PV was suppressed by Tween 20 in a dose-dependent manner in both emulsion system using AMVN and AAPH, and the tendency of suppression was clearer in the system using AMVN than in that using AAPH. Contrarily, there were no significant differences in PV when emulsions were prepared with tocopherol-stripped oil. The particle size of emulsion droplets were also consistent among emulsions prepared. However, tocopherol contents at lipid phase of the emulsions prior to oxidation decreased with concentration of Tween 20 . These results suggest that excess Tween 20 could cause tocopherol to migrate from the lipid phase of the emulsions to lipid-oil interface, suppressing the oxidation of emulsions.
\end{abstract}

\section{Keywords}

O/W Emulsion, Oxidation, Tween 20

\section{Introduction}

Oxidative deterioration of foods containing oils and fats may be affected by light, temperature, metals, or peroxide contained in food. Many studies to suppress oxidation of oils and fats have been conducted. Usually, oxidation of oils and fats 
is affected by its composition of fatty acids: oils and fats having unsaturated fatty acids, such as linoleic acid (C18:2) or linolenic acid (C18:3) are more sensitive to oxidation than that having saturated fatty acids such as palmitic acid (C16:0) or stearic acid (C18:0). To prevent food containing oils and fats from oxidation, a lot of antioxidants were evaluated its antioxidative activity [1].

In most cases, oils and fats are present as emulsions, such as water-in-oil $(\mathrm{W} / \mathrm{O})$ emulsion (e.g. margarine and butter) or oil-in-water $(\mathrm{O} / \mathrm{W})$ emulsion (e.g. beverages, milk, and fresh cream). In addition, it is known that the oxidative stability of lipid particles in $\mathrm{O} / \mathrm{W}$ emulsions is influenced by many factors, such as $\mathrm{pH}$ or types of emulsifiers composing the droplet interface [2]. Because lipid oxidation in $\mathrm{O} / \mathrm{W}$ emulsions take places mostly at the interface of lipid and aqueous phase, the characteristics of the interface of dispersed particles could have a large effect on the oxidative stability of oils and fats in O/W emulsions [3] [4].

Usually, an emulsifier is essential for preparing emulsions, except for particular cases, such as "three-phase emulsion" [5] [6]. Because it is important for many foods and cosmetics to stabilize emulsions or emulsion particle, most research has been focused on the physical/physicochemical properties of emulsifiers in order to understand the effect of $\mathrm{pH}$, ionic strength, and temperature on physical and chemical stability of emulsions [7], understand effect of carrier oil and emulsifier type on phase behavior and physical stability [8], or prepare $\mathrm{pH}$-responsive emulsions [9]. On the contrary, the relationship between emulsifier and oxidation of carrier oil has been also researched. For example, Hu et al. reported that oxidation of corn oil-emulsion was suppressed by adding casein as emulsifier, perhaps because casein produces thick layers on emulsion or it possesses chelating properties [10]. Lee et al. reported that phosphatidylcholine decreased the oxidation of sunflower oil-emulsion due to the ability of phosphatidylcholine to quench singlet oxygen, a trigger of oxidation [11]. In these researches, casein and phospholipids showed intrinsic antioxidant activity. However, few reports have previously mentioned that an emulsifier affects the activity of antioxidant in emulsions.

Kiralan et al. demonstrated that an emulsifier, such as Tween 20, has the ability to solubilize antioxidants, such as tocopherols, to the water phase of the emulsion, which resulted in a low degree of oxidation of the emulsion prepared from soy bean oil [12]. Our previous report also demonstrated that some additional emulsifier can dramatically increase the activity of lyophobic antioxidants such as catechin in $\mathrm{O} / \mathrm{W}$ emulsion prepared from milk fat [13]. We thought that some emulsifier could modify the physical location of antioxidants to oil-water interface where oxidation occurs. Because the oxidation behavior of an emulsion varies with its composition, such as the oils and emulsifiers used, the concentration of emulsifier, or the ratio of oil to water, further validation is required.

In this study, the effect of emulsifier concentration on the oxidation of an $\mathrm{O} / \mathrm{W}$ emulsion prepared from canola oil was investigated. 


\section{Experimental Procedures}

\subsection{Materials}

\subsubsection{Oils}

Canola oil (Nisshin OilliO Group, Ltd., Tokyo, Japan) was purchased from a grocery supermarket in Shobara, Japan. This oil contained $\sim 400$ ppm of tocopherol (varying slightly among individual products.). The fatty acid composition of the canola oil used is summarized in Table 1 . The result was the same as other report [14].

\subsubsection{Emulsifiers and Chemicals}

Tween 20 was purchased from Tokyo Chemical Industry Co., Ltd. (Tokyo, Japan). Decaglyceryl monomyristate was donated from Taiyo Kagaku Co., Ltd. (Yokkaichi, Mie, Japan). 2,2'-azobis (2,4-dimethylaleronitrile) (AMVN) and 2,2'-azobis-2-methyl-propanimidamide dihydrochloride amide dihydrochloride (AAPH) were purchased from Tokyo Chemical Industry Co., Ltd. to act as radical generators. Activated charcoal (powder) was obtained from Wako Pure Chemical Industries, Ltd. (Osaka, Japan). Other reagents and solvents used in this study were of analysis grade.

\subsection{Methods}

\subsubsection{Purification of Oil (To Remove Tocopherol)}

Canola oil ( $60 \mathrm{~g}$ ) was dissolved by $300 \mathrm{~mL}$ of hexane flowed by adding activated charcoal. After $15 \mathrm{~min}$ of agitation, filtrate was obtained by suction filtration using Kiriyama funnel. Filtrate was filtered with filter paper (Advantec ${ }^{\circledR}$ No.1) again. Purified oil was obtained after evaporation of solvent.

\subsubsection{Preparation of $0 / \mathrm{W}$ Emulsion}

Ultrapure water $(70 \mathrm{~mL})$ containing Tween 20 or decaglyceryl monomyristate was added to canola oil (30 g). This was then emulsified using homogenizer (IKA ${ }^{\oplus}$ T10 basic, ULTRA-TURRAX ${ }^{\oplus}$, Jahnke und Kunkel GmbH, Staufen, Germany) at 8,000 rpm for $3 \mathrm{~min}$. Concentrations of emulsifiers used were 0.5, 2.5, 5.0 , and $10 \mathrm{wt} \%$ vs oil. This emulsion contained $0.2 \mathrm{wt} \%$ of AMVN and AAPH vs oil. AMVN and AAPH were dissolved with oil and water, respectively, prior to homogenization.

\subsubsection{Oxidation Test}

Emulsions were incubated under moderate agitation at $34^{\circ} \mathrm{C}$ in the dark for 7

Table 1. Fatty acid compositions of canola oil used in this study.

\begin{tabular}{ccc}
\hline Palmitic acid & $(\mathrm{C} 16: 0)$ & $4.0 \pm 0.1$ \\
Stearic acid & $(\mathrm{C} 18: 0)$ & $1.8 \pm 0.2$ \\
Oleic acid & $(\mathrm{C} 18: 1)$ & $59.9 \pm 1.0$ \\
Linoleic acid & $(\mathrm{C} 18: 2)$ & $19.7 \pm 0.4$ \\
Linolenic acid & $(\mathrm{C} 18: 3)$ & $8.7 \pm 0.3$ \\
others & & $6.2 \pm 1.3$ \\
\hline
\end{tabular}


days. After incubation, oil was extracted and peroxide value $(\mathrm{mEq} / \mathrm{kg})($ section 2.2.6) and content of tocopherol (section 2.2.7) were monitored.

Particle size (section 2.2.8) and content of tocopherol in lipid phase (section 2.2.9) were also measured as necessary.

\subsubsection{Fatty Acid Composition of Canola Oil}

Fatty acids of canola oil was methyl-esterified using hydrochloric acid in methanol, as described by Jham et al. [15], and subjected to gas chromatography (GC) using a GC-17A (Shimadzu, Kyoto, Japan) equipped with a flame ionization detector and a fused silica capillary column, DB-WAX $(0.25 \mathrm{~mm} \times 60 \mathrm{~m}$, $0.25 \mu \mathrm{m}$; Agilent Technologies, CA, USA). The temperature of both the injector and detector were $250^{\circ} \mathrm{C}$. Helium at $80 \mathrm{kPa}$ was used as the carrier gas. The column temperature was $190^{\circ} \mathrm{C}$.

\subsubsection{Extraction of Oil from Emulsion}

Emulsion $(3.3 \mathrm{~mL})$ was drawn into a test tube and mixed with mixture of chloroform-methanol (2:1, v/v), followed by centrifugation at $3000 \mathrm{rpm}$ for $5 \mathrm{~min}$. Then, a lower layer was carefully drawn by pasteur pipette. Oil was obtained after evaporation of the solvent and its peroxide value (Section 2.2.6) and tocopherol content (Section 2.2.7) were analyzed.

\subsubsection{Peroxide Value (PV, $\mathrm{mEq} / \mathrm{kg}$ )}

Potentiometric titrations of peroxide value $(\mathrm{PV}, \mathrm{meq} / \mathrm{kg})$ of the oil were performed in accordance with the standard method for the analysis of fats, oils and related materials as prescribed by the Japan Oil Chemists' Society [16].

\subsubsection{Quantification of Tocopherol in Oil}

Tocopherol content of oil was analyzed by high performance liquid chromatography (HPLC). The HPLC system consisted of a Waters 2695 Separations module and a Waters 2487 Dual $\lambda$ Absorbance Detector. A Shodex 5SIL-4E (4.6 x 250 $\mathrm{mm}, 5 \mu \mathrm{m}$, Showa Denko KK, Tokyo, Japan) column was used. The mobile phase was composed of a hexane-acetonitrile mixture (200:1, v/v). The flow rate was $1.0 \mathrm{~mL} / \mathrm{min}$, and the column temperature was $30^{\circ} \mathrm{C}$. Elutes were detected at $300 \mathrm{~nm}$. 2,2,5,7,8-pentamethyl-6-chromanol (PMC) was used as an internal standard for quantification of tocopherols and the following equations of calibration curves based on authentic compounds were used: $\alpha$-Toc, $\mathrm{y}=0.5428 \mathrm{x}+$ $0.0295\left(\mathrm{R}^{2}=0.9292\right) ; \beta$-Toc, $\mathrm{y}=0.5208+0.1226\left(\mathrm{R}^{2}=0.9590\right) ; \gamma-$ Toc, $\mathrm{y}=0.75 \mathrm{x}$ $+0.0742\left(R^{2}=0.9607\right) ; \gamma$-Toc, $y=0.7327 x-0.0477\left(R^{2}=0.9767\right)$. Each tocopherol and PMC was purchased from Mitsubishi-Chemical Foods Corporation (Tokyo, Japan).

\subsubsection{Particle Size of Emulsion}

Particle size of emulsion was measured using an inverted optical microscope (200x), and calculated with "Image J" software (National Institute of Mental Health, Bethesda, MA). For the calculation, 50 particles were randomly picked up. 


\subsubsection{Tocopherol Content of Lipid Phase}

Emulsion $(1 \mathrm{~mL})$ was drawn into a micro tube and centrifuged at 15,000 rpm for $1 \mathrm{~h}$. The upper (oil) layer $(300 \mu \mathrm{L})$ was collected and added to a mixture of chloroform-methanol-water (10:5:3, v/v/v), followed by centrifugation at $3000 \mathrm{rpm}$ for $5 \mathrm{~min}$. Then, the lower layer was collected and dried by nitrogen stream. The tocopherol content of the oil obtained was measured as described in section 2.2.7.

\subsubsection{Statistical Analysis}

All experiments were conducted in, at least, triplicate. Data are shown as the means \pm standard deviation. A significant difference was determined by Scheffe's test $(\mathrm{P}<0.01)$.

\section{Results and Discussion}

\subsection{PV and Tocopherol Content on Oxidation Test}

First, O/W emulsions were prepared with Tween 20 as emulsifier, while AMVN was used as a radical generator to promote oxidation. At any concentration of Tween 20, PV increased with incubation time, and reached PV $25-35 \mathrm{mEq} / \mathrm{kg}$ at 7 days of incubation (Figure 1(a)). Although there were no significant differences, a tendency towards PV suppression was observed with concentration of Tween 20. Especially, at initial stage when PV was under $\sim 25 \mathrm{mEq} / \mathrm{kg}$, the tendency was clearer. On the contrary, there were no tendency relating the concentration of Tween 20 and residual tocopherol (\%) in lipid phase of the emulsion (Figure 1(b)).

Then, the days when PV reached at $20 \mathrm{mEq} / \mathrm{kg}$ were calculated from the fitting curve below.

$$
\begin{gathered}
y=-0.3039 x^{2}+6.4422 x+0.6459\left(R^{2}=0.9801,0.5 \%\right) \\
y=-0.033 x^{2}+4.4752 x+2.1114\left(R^{2}=0.9301,2.5 \%\right)
\end{gathered}
$$

(a)

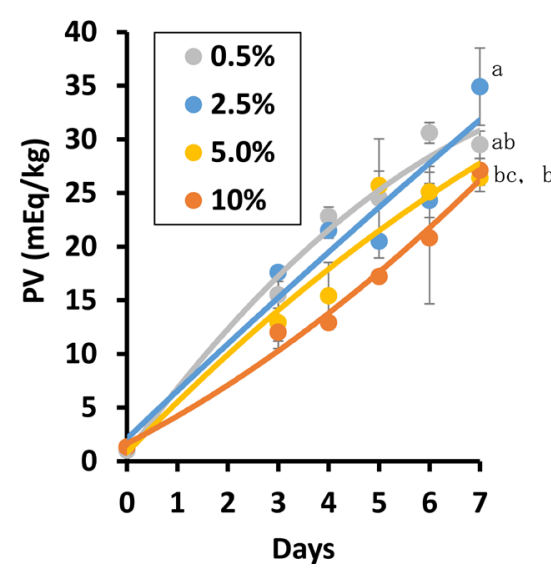

(b)

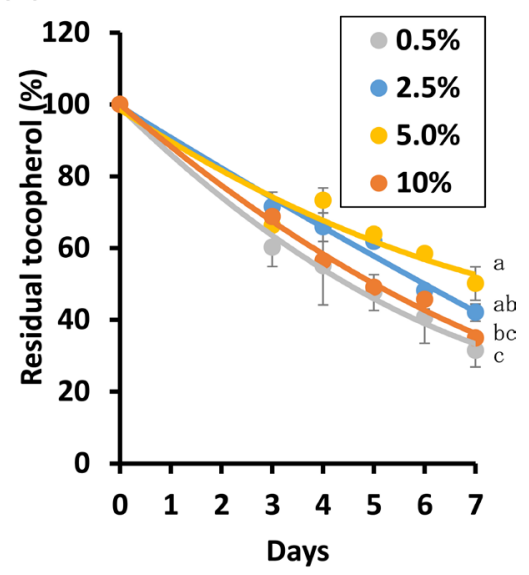

Figure 1. Time course of PV (mEq/kg) (a), and residual tocopherol (\%) (b) in emulsions prepared by different emulsifier contents. a-c: significantly different among different letters $(\mathrm{p}<0.05$, Scheffe's test). Data were shown as mean \pm standard deviation $(n=3)$. 


$$
\begin{gathered}
y=-0.1421 x^{2}+4.8456 x+0.8083\left(R^{2}=0.9437,5.0 \%\right) \\
y=0.1574 x^{2}+2.4076 x+1.6433\left(R^{2}=0.9851,10 \%\right)
\end{gathered}
$$

Data obtained by substituting $y=20$ in each equation were summarized in Table 2. Data obtained from the emulsion oxidized with AAPH in the same way were also shown. Although it was not a clear as that of AMVN, similar tendency was observed from the emulsion oxidized with AAPH.

When emulsions were prepared with purified (i.e. tocopherol-free) canola oil and oxidized with AMVN, PVs of all emulsions with different concentration of Tween 20 increased very rapidly and reached $\sim 100 \mathrm{mEq} / \mathrm{kg}$ within the first day (Table 3). There were no significant differences. This result means that tween 20 does not affect oxidation of oil in droplets themselves.

These results, suggest that Tween 20 affected the oxidation stability of the emulsion prepared in this study, and that with the addition of the more Tween 20 , there was greater suppression of the oxidation of the emulsion. Tween 20 may have some influence on tocopherol existing in lipid phase in emulsion droplets.

\subsection{Diameter of Emulsion Droplets and Tocopherol Content at Lipid Phase}

To research the effect of Tween 20 more clearly, the diameter of emulsion droplets and tocopherol content existing in lipid phase before oxidation of emulsion were measured.

Figure 2 showed the average diameter of emulsion droplets obtained from emulsions with different concentration of Tween 20 at the start of the experiment and after 7 days. On the first day, average diameter was almost the same among emulsions containing different concentration of Tween 20. The average diameter of each emulsion after 7 days was larger than on the first 0 day, owing to deterioration of each emulsion. This is because unstable emulsion droplets

Table 2. The days when PV reached at $20 \mathrm{mEq} / \mathrm{kg}$.

\begin{tabular}{ccc}
\hline Tween 20 (\%) & AMVN & AAPH \\
\hline 0.5 & 3.6 & 2.7 \\
2.5 & 4.1 & 2.9 \\
5.0 & 4.6 & 3.9 \\
10 & 5.6 & 4.0 \\
\hline
\end{tabular}

Table 3. PVs of emulsions prepared with purified oil on the first day of oxidation period.

\begin{tabular}{cc}
\hline Tween $20(\%)$ & PV $(\mathrm{mEq} / \mathrm{kg})$ \\
\hline 0.5 & $97.9 \pm 08.10$ \\
2.5 & $128.6 \pm 32.5$ \\
5.0 & $108.7 \pm 6.70$ \\
10 & $133.1 \pm 31.2$ \\
\hline
\end{tabular}




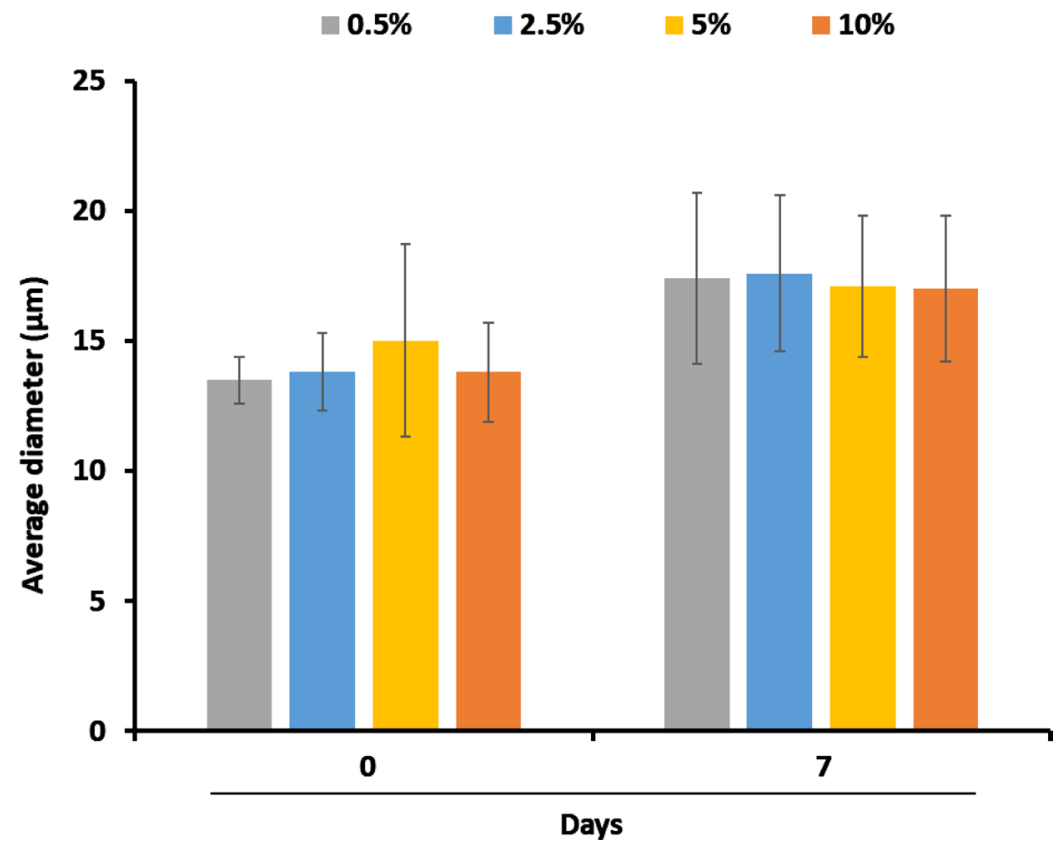

Figure 2. Average diameter of emulsion droplets obtained from emulsions with different concentration of Tween 20 .

partially coalesce, resulting in larger emulsion droplets on average [17] [18].

Emulsion droplets have a crucial effect on the oxidation stability of an emulsion. Because smaller (lesser-volume) droplets have more specific surface area than larger droplets, emulsions containing a smaller average droplet size generally show low stability against oxidation than the larger droplets [19]. This result suggests that oxidative potential based on the size of emulsion droplets was the same among emulsions despite having different concentration of Tween 20.

Finally, tocopherol content in the lipid phase of emulsion on the first day was measured. This revealed that tocopherol content in the lipid phase decreased with the concentration of Tween 20 , although the difference was not statistically significant (Figure 3). This result suggested that tocopherol content, not only in water phase, but also in oil-water interphase of the emulsions, was increased by increasing the concentration of Tween 20. Because oxidation of emulsion occurs mainly at the oil-water inter phase, it was shown that the antioxidant efficacy of tocopherol was modified by changing its localization in the emulsion droplet by increasing the concentration of Tween 20. However, the effect of modifying the antioxidant efficacy was modest (Figure 1(a)). In addition, emulsions prepared with decaglyceryl monomyristate as emulsifier containing AMVN as radical generator, showed no tendency in terms of the concentration of decaglyceryl monomyristate and PV (data not shown). Richards et al. also reported that excess Brij, an emulsifier, could cause propyl gallate to migrate from lipid phase to the water phase in $\mathrm{O} / \mathrm{W}$ emulsion using salmon oil, with no effect on its antioxidant efficacy [20].

Contrarily, Panya et al. reported that antioxidant efficacy of rosmarinate alkyl esters in $\mathrm{O} / \mathrm{W}$ emulsion was increased by increasing the concentration of Tween 20 [21]. Kiralan et al. also reported that clear modification of antioxidant efficacy 


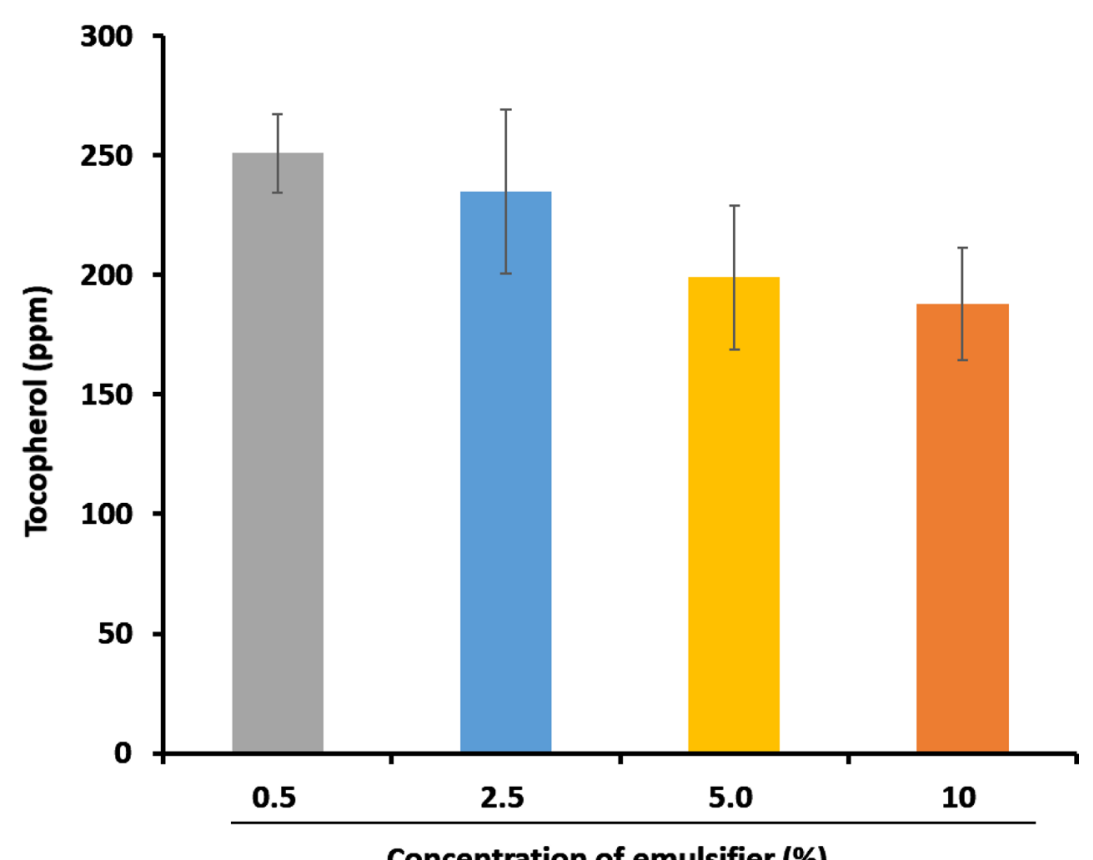

Figure 3. Tocopherol content in the lipid phase of emulsion on the first day.

of $\alpha / \delta$-tocopherol was shown by increasing the concentration of Tween 20 [12]. In both experiments, emulsions were prepared from soybean oil with an oil/water ratio of 1:99 (w/w), and Tween 20 was added at $1 \mathrm{wt} \%$ vs oil, and additional Tween 20 incorporated at $0.1 \%-2.5 \%$. From these reports, it was suggested that the effect of additional/excess emulsifier differed with the composition of the emulsion, such as emulsifier used, antioxidants used, ratio of oil/water, and emulsions used.

\section{Conclusion}

If oxidation of oils and fats in emulsion can suppress by using emulsifier, economic impact is big because most of emulsifiers are relatively cheaper than most of antioxidant compounds. In this study, using a simple O/W emulsion system composed of canola oil, water, and emulsifier, the effect of emulsifier on oxidation of emulsion was investigated. Although the effect was modest, excess amount of Tween 20 modified the antioxidant efficacy of tocopherol in a dose-dependent manner. The speculation is that Tween 20 can help tocopherol to migrate from the lipid phase to the oil-water interphase, where the oxidation occurs. Further research to define the optimum combination of oil, water, and emulsifier is required.

\section{Acknowledgements}

We would like to thank Editage (www.editage.jp) for English language editing.

\section{References}

[1] Frankel, E.N. (1993) In Search of Better Methods to Evaluate Natural Antioxidants 
and Oxidative Stability in Food Lipids. Trends in Food Science \& Technology, 4, 220-225. https://doi.org/10.1016/0924-2244(93)90155-4

[2] McClements, D.J. and Decker, E.A. (2000) Lipid Oxidation in Oil-in-Water Emulsions: Impact of Molecular Environment on Chemical Reactions in Heterogeneous Food System. Journal of Food Science, 65, 1270-1282.

https://doi.org/10.1111/j.1365-2621.2000.tb10596.x

[3] Schwarz, K., Huang, S.W., German, J.B., Tiersch, B., Hartmann, J. and Frankel, E.N. (2000) Activities of Antioxidants Are Affected by Colloidal Properties of Oil-in-Water and Water-in-Oil Emulsions and Bulk Oils. Journal of Agricultural and Food Chemistry, 48, 4874-4882. https://doi.org/10.1021/jf991289a

[4] Hu, M., McClements, D.J. and Decker, E.A. (2003) Lipid Oxidation in Corn Oil-in-Water Emulsions Stabilized by Casein, Whey Protein Isolate, and Soy Protein Isolate. Journal of Agricultural and Food Chemistry, 51, 1696-1700. https://doi.org/10.1021/jf020952j

[5] Tajima, K., Imai, Y., Nakamura, A. and Koshinuma, M. (2001) Three-Phase Emulsions of Hexadecane with Dimyristoylphosphatidylglycerol Sodium Salt in Water: An Interpretation by New Phase Transition in Bilayer Assembly. Journal of Oleo Science, 50, 475-484. https://doi.org/10.5650/jos.50.475

[6] Dickinson, E. (2010) Food Emulsions and Foams: Stabilization by Particles. Current Opinion in Colloid \& Interface Science, 15, 40-49. https://doi.org/10.1016/j.cocis.2009.11.001

[7] Cheng, Q., Eric, A.D., Hang, X. and David, J.M. (2012) Physical and Chemical Stability of $\beta$-Carotene-Enriched Nanoemulsions: Influence of $\mathrm{pH}$, Ionic Strength, Temperature, and Emulsifier Type. Food Chemistry, 132, 1221-1229. https://doi.org/10.1016/j.foodchem.2011.11.091

[8] Cheng, Q., Eric, A.D., Hang, X. and David, J.M. (2012) Solid Lipid Nanoparticles: Effect of Carrier Oil and Emulsifier Type on Phase Behavior and Physical Stability. Journal of the American Oil Chemists' Society, 89, 17-28. https://doi.org/10.1007/s11746-011-1882-0

[9] Raju, R.R., Liebig, F., Klemke, B. and Koetz, J. (2018) pH-Responsive Magnetic Pickering Janus Emulsions. Colloid and Polymer Science, 296, 1039-1046. https://doi.org/10.1007/s00396-018-4321-z

[10] Hu, M., McClements, D.J. and Decker, E.A. (2003) Lipid Oxidation in Corn Oil-in-Water Emulsions Stabilized by Casein, Whey Protein Isolate, and Soy Protein Isolate. Journal of Agricultural and Food Chemistry, 51, 1696-1700. https://doi.org/10.1021/jf020952j

[11] Lee, Y. and Choe, E. (2008) Singlet Oxygen Quenching Effects of Phosphatidylcholine in Emulsion Containing Sunflower Oil. Journal of Food Science, 73, 506-511. https://doi.org/10.1111/j.1750-3841.2008.00852.x

[12] Kiralan, S.S., Doğu-Baykut, E., Kittipongpittaya, K., McClements, D.J. and Decker, E.A. (2014) Increased Antioxidant Efficacy of Tocopherols by Surfactant Solubilization in Oil-in-Water Emulsions. Journal of Agricultural and Food Chemistry, 62, 10561-10566. https://doi.org/10.1021/jf503115j

[13] Yamamoto, Y., Hiyama, S., Takase, Y., Kadowaki, A. and Hara, S. (2014) Effects of Antioxidants and Additional Emulsifiers on the Stability of Emulsified Milk Fat in the Photo/Radical Oxidation System. Journal of Oleo Science, 63, 893-901. https://doi.org/10.5650/jos.ess14111

[14] Neff, W.E., Mounts, T.L., Rinsch, W.M., Konishi, H. and EI-Agaimy, M.A. (1994) Oxidative Stability of Purified Canola oil Triacylglycerols with Altered Fatty Acid 
Compositions as Affected by Triacylglycerol Composition and Structure. Journal of the American Oil Chemists' Society, 71, 1101-1109. https://doi.org/10.1007/BF02675903

[15] Jham, G.N., Teles, F.F.F. and Campos, L.G. (1982) Use of Aqueous $\mathrm{HCl} / \mathrm{MeOH}$ as Esterification Reagent for Analysis of Fatty Acids Derived from Soybean Lipids. Journal of the American Oil Chemists' Society, 59, 132-133. https://doi.org/10.1007/BF02662261

[16] Standard Method for the Analysis of Fats, Oils and Related Materials (Japan Oil Chem. Soc., ed), Peroxide Value (Potentiometric Method), 2.5.2.2-2013.

[17] Boode, K. and Walstra, P. (1993) Partial Coalescence in Oil-in-Water Emulsions 1. Nature of the Aggregation. Colloids and Surfaces A: Physicochemical and Engineering Aspects, 81, 121-137. https://doi.org/10.1016/0927-7757(93)80239-B

[18] Boode, K., Walstra, P. and de Groot-Mostert, A.E.A. (1993) Partial Coalescence in Oil-in-Water Emulsions 2. Influence of the Properties of the Fat. Colloids and Surfaces A: Physicochemical and Engineering Aspects, 81, 139-151. https://doi.org/10.1016/0927-7757(93)80240-F

[19] Lethuaut, L., Métro, F. and Genot, C. (2002) Effect of Droplet Size on Lipid Oxidation Rates of Oil-in-Water Emulsions Stabilized by Protein. Journal of the American Oil Chemists' Society, 79, 425-430. https://doi.org/10.1007/s11746-002-0500-z

[20] Richards, M.P., Chaiyasit, W., MaMlements, D.J. and Decker, E.A. (2002) Ability of Surfactant Micelles to Alter the Partitioning of Phenolic Antioxidants in Oil-in-Water Emulsions. Journal of Agricultural and Food Chemistry, 50, 1254-11259. https://doi.org/10.1021/jf011324p

[21] Panya, A., Laguerre, M., Bayrasy, C., Lecomte, J., Villeneuve, P., McClements, D.J. and Decker, E.A. (2011) An Investigation of the Versatile Antioxidant Mechanisms of Action of Rosmarinate Alkyl Esters in Oil-in-Water Emulsions. Journal of Agricultural and Food Chemistry, 60, 2692-2700. https://doi.org/10.1021/jf204848b 